\title{
Detecting Changing Polarization Structures in Sagittarius A* With High Frequency Vlbi
}

\section{Citation}

Fish, Vincent L., Sheperd S. Doeleman, Avery E. Broderick, Abraham Loeb, and Alan E. E. Rogers. 2009. "DETECTING CHANGING POLARIZATION STRUCTURES IN SAGITTARIUS A* WITH HIGH FREQUENCY VLBI." The Astrophysical Journal 706 (2): 1353-63. https:// doi.org/10.1088/0004-637x/706/2/1353.

\section{Permanent link}

http://nrs.harvard.edu/urn-3:HUL.InstRepos:41393462

\section{Terms of Use}

This article was downloaded from Harvard University's DASH repository, and is made available under the terms and conditions applicable to Other Posted Material, as set forth at http:// nrs.harvard.edu/urn-3:HUL.InstRepos:dash.current.terms-of-use\#LAA

\section{Share Your Story}

The Harvard community has made this article openly available. Please share how this access benefits you. Submit a story.

Accessibility 


\title{
DETECTING CHANGING POLARIZATION STRUCTURES IN SAGITTARIUS A* WITH HIGH FREQUENCY
} VLBI

\author{
Vincent L. Fish ${ }^{1}$, Sheperd S. Doeleman ${ }^{1}$, Avery E. Broderick ${ }^{2}$, Abraham Loeb ${ }^{3}$, and Alan E. E. Rogers ${ }^{1}$ \\ ${ }^{1}$ Massachusetts Institute of Technology, Haystack Observatory, Route 40, Westford, MA 01886, USA \\ ${ }^{2}$ Canadian Institute for Theoretical Astrophysics, University of Toronto, 60 St. George Street, Toronto, ON M5S 3H8, Canada \\ ${ }^{3}$ Institute for Theory and Computation, Harvard University, Center for Astrophysics, 60 Garden Street, Cambridge, MA 02138, USA \\ Received 2009 June 29; accepted 2009 October 16; published 2009 November 11
}

\begin{abstract}
Sagittarius $\mathrm{A}^{*}$ is the source of near infrared, X-ray, radio, and (sub)millimeter emission associated with the supermassive black hole at the Galactic Center. In the submillimeter regime, Sgr A* exhibits time-variable linear polarization on timescales corresponding to $<10$ Schwarzschild radii of the presumed $4 \times 10^{6} M_{\odot}$ black hole. In previous work, we demonstrated the potential for total-intensity (sub)millimeter-wavelength very long baseline interferometry (VLBI) to detect time-variable-and periodic - source structure changes in the Sgr A* black hole system using nonimaging analyses. Here, we extend this work to include full polarimetric VLBI observations. We simulate full-polarization (sub)millimeter VLBI data of Sgr A* using a hot spot model that is embedded within an accretion disk, with emphasis on nonimaging polarimetric data products that are robust against calibration errors. Although the source-integrated linear polarization fraction in the models is typically only a few percent, the linear polarization fraction on small angular scales can be much higher, enabling the detection of changes in the polarimetric structure of Sgr $\mathrm{A}^{*}$ on a wide variety of baselines. The shortest baselines track the source-integrated linear polarization fraction, while longer baselines are sensitive to polarization substructures that are beam-diluted by connected-element interferometry. The detection of periodic variability in source polarization should not be significantly affected even if instrumental polarization terms cannot be calibrated out. As more antennas are included in the (sub)millimeter-VLBI array, observations with full polarization will provide important new diagnostics to help disentangle intrinsic source polarization from Faraday rotation effects in the accretion and outflow region close to the black hole event horizon.
\end{abstract}

Key words: accretion, accretion disks - black hole physics - Galaxy: center - polarization - submillimeter techniques: interferometric

Online-only material: color figures

\section{INTRODUCTION}

The Galactic Center source Sagittarius A* (Sgr A*) provides the best case for high-resolution, detailed observations of the accretion and outflow region surrounding the event horizon of a black hole. There are several compelling reasons to observe Sgr A* with very long baseline interferometry (VLBI) at (sub)millimeter ${ }^{4}$ wavelengths. The spectrum of Sgr $\mathrm{A}^{*}$ peaks in the millimeter (Markoff et al. 2007, and references therein). Interstellar scattering, which varies as the wavelength $\lambda^{2}$, becomes less than the fringe spacing of the longest baseline available to VLBI in the millimeter-wavelength regime. Indeed, VLBI on the longest baselines available at $345 \mathrm{GHz}$ probes scales of twice the Schwarzschild radius $\left(R_{\mathrm{S}}\right)$ for a $4 \times 10^{6} M_{\odot}$ black hole. From previous observations at $230 \mathrm{GHz}$, it is known that there are structures on scales smaller than a few $R_{\mathrm{S}}$ (Doeleman et al. 2008). Such high angular resolution, presently unattainable by any other method (including facility instruments such as the Very Long Baseline Array), is necessary to match the expected spatial scales of the emitting plasma in the innermost regions surrounding the black hole and will be required to unambiguously determine the inflow/outflow morphology and permit tests of general relativity.

This sensitivity to small spatial scales also makes millimeter polarimetric VLBI possible. Although the linear polarization

\footnotetext{
4 We shall henceforth use the term "millimeter" to denote wavelengths of $1.3 \mathrm{~mm}$ of shorter (in contrast with observations at $3 \mathrm{~mm}$ and $7 \mathrm{~mm}$, which are sometimes also referred to as "millimeter" wavelengths).
}

fraction of Sgr $\mathrm{A}^{*}$ integrated over the entire source is only a few percent (e.g., Marrone et al. 2007), the fractional polarization on small angular scales is likely much larger. In general, relativistic accretion flow models predict that the electric vector polarization angle (EVPA) will vary along the circumference of the accretion disk (Bromley et al. 2001; Broderick \& Loeb 2005, 2006), indicating that single-dish observations and connected-element interferometers probably underestimate linear polarization fractions due to beam depolarization.

Polarized synchrotron radiation coming from $\mathrm{Sgr} \mathrm{A}^{*}$ was detected by Aitken et al. (2000) at millimeter and submillimeter wavelengths. Multiple observations since then have demonstrated that the polarized emission is variable on timescales from hours to many days (Bower et al. 2005; Macquart et al. 2006; Marrone et al. 2006a, 2007, 2008). In one case, the timescale of variability and the trace of polarization in the Stokes $(Q, U)$ plane of the millimeter-wavelength emission are suggestive of the detection of an orbit of a polarized blob of material (Marrone et al. 2006b). Near infrared observations by Trippe et al. (2007) are also consistent with a hot spot origin for periodic variability. It is possible that connected-element interferometry may suffice to demonstrate polarization periodicity, but millimeterwavelength VLBI, which effectively acts as a spatial filter on scales of a few to a few hundred $R_{\mathrm{S}}$, can be more sensitive to changing polarization structures.

Initial millimeter VLBI observations of Sgr A* will necessarily utilize nonimaging analysis techniques, for reasons outlined in Doeleman et al. (2009a, henceforth Paper I). One way 
to do this is to analyze the so-called interferometric "closure quantities," which are relatively immune to calibration errors (Rogers et al. 1974, 1995). In Paper I, we considered prospects for detecting the periodicity signature of a hot spot orbiting the black hole in Sgr A* via closure quantities in total-intensity millimeter-wavelength VLBI. In the single polarization case, it is necessary to construct closure quantities from at least three or four antennas in order to produce robust observables, since the timescales of atmospheric coherence and frequency standard stability do not permit standard nodding calibration techniques. Closure quantities can be used in full-polarization observations as well, but it is also possible to construct robust observables on a baseline of two antennas by taking visibility ratios between different correlation products. In this work, we extend our techniques to explore polarimetric signatures of a variable source structure in $\mathrm{Sgr} \mathrm{A}^{*}$, with emphasis on ratios of baseline visibilities.

\section{MODELS AND METHODS}

We employ the same models discussed in Paper I to describe the flaring emission of Sgr A*, and shall only briefly review these here, directing the reader to Paper I, and references therein, for more detail. These models consist of an orbiting hot spot, modeled by a Gaussian overdensity of power-law electrons, embedded in a radiatively inefficient accretion flow, containing both thermal and nonthermal electron populations.

The primary emission mechanism for both components is synchrotron. We model the emission from the thermal and nonthermal electrons using the emissivities described in Yuan et al. (2003) and Jones \& O'Dell (1977), respectively, appropriately modified to account for relativistic effects (see Broderick \& Blandford 2004 for a more complete description of polarized general relativistic radiative transfer). Since we necessarily are performing the fully polarized radiative transfer, for the thermal electrons we employ the polarization fraction derived in Petrosian \& McTiernan (1983). In doing so we have implicitly assumed that the emission due to the thermal electrons is locally isotropic, which, while generally not the case in the presence of ordered magnetic fields, is unlikely to modify our results significantly. For both electron populations the absorption coefficients are determined directly via Kirchoff's law.

As described in Paper I, the assumed magnetic field geometry was toroidal, consistent with simulations and analytical expectations for magnetic fields in accretion disks, though other field configurations are possible (e.g., Huang et al. 2009). While the overall flux of our models is relatively insensitive to the magnetic field geometry, the polarization is dependent on it. However, polarization light curves and maps with considerably different magnetic field geometries (e.g., poloidal) are qualitatively similar, showing large swings in polarization angle and patches of nearly uniform polarization in the images.

Generally, synchrotron emission has both linearly and circularly polarized components. However, the circular polarization fraction is suppressed by an additional factor of the electron Lorentz factor. For the electrons producing the millimeter emission, this corresponds to a reduction by a factor of 30-100 in Stokes $V$ in comparison to Stokes $Q$ and $U$. This is consistent with observations by Marrone et al. (2006a), who obtain an upper limit of $\sim 1 \%$ circular polarization at $340 \mathrm{GHz}$. Therefore, we explicitly omitted the circular polarization terms in the computation of flaring polarization.

In addition, we have neglected the potentially modest intrinsic Faraday rotation. Within $r \lesssim 10^{2}-10^{3} R_{S}$ the accret-

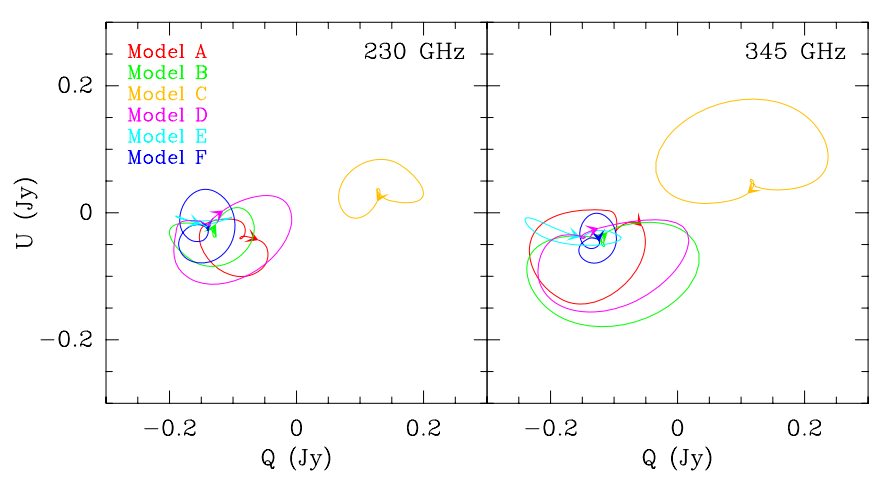

Figure 1. Integrated polarization traces of the models in the Stokes $(Q, U)$ plane at 230 and $345 \mathrm{GHz}$ over a full hot spot orbit, as would be seen by the SMA (for instance).

(A color version of this figure is available in the online journal.)

ing electrons are expected to be substantially relativistic, and thus not contribute significantly to the rotation measure within the millimeter-emitting region. This is consistent with the lack of observed Faraday depolarization at these wavelengths (e.g., Aitken et al. 2000; Marrone et al. 2007), which itself implies the absence of significant in situ Faraday rotation. Similarly, beam depolarization caused by variations within an external Faraday screen on angular scales comparable to that of the emission region are empirically excluded. This leaves the possibility of a smoothly varying external Faraday screen, which manifests itself in the VLBI data as an additional phase difference between right and left circularly polarized visibilities, but does not affect our analysis otherwise.

Model images are created in each of the Stokes parameters $I, Q$, and $U$. Six models differing in hot spot orbital period, black hole spin, and accretion disk inclination and majoraxis orientation are produced at 230 and $345 \mathrm{GHz}$, as in Paper I. Model properties are summarized in Table 1. Sourceintegrated linear polarization fractions range from $0.8 \%$ to $26 \%$ for models including both a disk and a hot spot, depending on the model and hot spot orbital phase, with typical integrated quiescent polarization fractions (of the disk alone) of $10 \%-$ $15 \%$. Integrated EVPA variation over the course of the hot spot orbit ranges from $4^{\circ}$ to $57^{\circ}$, depending on the model. The integrated polarization fractions and EVPA variations as well as the polarization traces in the Stokes $(Q, U)$ plane (Figure 1) are all broadly consistent with the range of variability seen in the Submillimeter Array (SMA) observations reported by Marrone et al. (2006b). The local linear polarization fraction can be much higher, exceeding $70 \%$ in some parts of the accretion disk.

Simulated array data are produced by the Astronomical Image Processing System (AIPS) task UVCON for each of the Stokes parameters. The array is taken to consist of up to seven stations: the Caltech Submillimeter Observatory, James Clerk Maxwell Telescope, and six SMA telescopes phased together into a single station (Hawaii); the Arizona Radio Observatory Submillimeter Telescope (SMT); a phased array consisting of eight telescopes in the Combined Array for Research in Millimeter-wave Astronomy (CARMA); the Large Millimeter Telescope (LMT); the $30 \mathrm{~m}$ Institut de radioastronomie millimétrique dish at Pico Veleta (PV); the Plateau de Bure Interferometer phased together as a single station $(\mathrm{PdB})$; and a site in Chile, either a single 10 or $12 \mathrm{~m}$ class telescope (Chile 1 ) or a phased array of 10 dishes of the Atacama Large Millimeter Array (Chile 10). Details of the method as well as assumed parameters of the telescopes are given in Paper I. 
Table 1

Model Parameters

\begin{tabular}{|c|c|c|c|c|c|c|c|c|c|c|c|c|}
\hline Model & $\begin{array}{c}a \\
\left(R_{G}\right)^{\mathrm{a}}\end{array}$ & $\begin{array}{c}\text { Period } \\
\text { (minutes) }\end{array}$ & $\begin{array}{c}i \\
\left({ }^{\circ}\right) \\
\end{array}$ & $\begin{array}{c}\mathrm{PA}^{\mathrm{b}} \\
\left({ }^{\circ}\right)\end{array}$ & $\begin{array}{c}v \\
(\mathrm{GHz})\end{array}$ & $\begin{array}{c}\text { Disk Flux } \\
(\mathrm{Jy})\end{array}$ & $\begin{array}{c}\text { Min Flux } \\
(\mathrm{Jy})\end{array}$ & $\begin{array}{c}\text { Max Flux } \\
(\mathrm{Jy})\end{array}$ & $\begin{array}{c}\text { Disk Pol. }^{\mathrm{d}} \\
(\%)\end{array}$ & $\begin{array}{c}\text { Disk EVPA } \\
\left({ }^{\circ}\right)\end{array}$ & $\begin{array}{c}\text { Min Pol. }^{\mathrm{d}} \\
(\%)\end{array}$ & $\begin{array}{c}\text { Max Pol. }^{\mathrm{d}} \\
(\%)\end{array}$ \\
\hline \multirow[t]{2}{*}{ A } & 0 & 27.0 & 30 & 90 & 230 & 3.19 & 3.49 & 4.05 & 10 & -75 & 7.0 & 16 \\
\hline & & & & & 345 & 3.36 & 3.63 & 5.28 & 11 & -81 & 6.0 & 24 \\
\hline \multirow[t]{2}{*}{ B } & 0 & 27.0 & 60 & 90 & 230 & 3.03 & 3.05 & 4.03 & 14 & -84 & 6.9 & 20 \\
\hline & & & & & 345 & 2.96 & 2.99 & 4.78 & 13 & -80 & 2.2 & 26 \\
\hline \multirow[t]{2}{*}{$\mathrm{C}$} & 0 & 27.0 & 60 & 0 & 230 & 3.03 & 3.05 & 4.03 & 14 & 6 & 6.9 & 20 \\
\hline & & & & & 345 & 2.96 & 2.99 & 4.78 & 13 & 10 & 2.2 & 26 \\
\hline \multirow[t]{2}{*}{ D } & 0.9 & 27.0 & 60 & 90 & 230 & 2.98 & 2.99 & 4.05 & 15 & -86 & 0.8 & 21 \\
\hline & & & & & 345 & 2.96 & 2.97 & 4.00 & 15 & -82 & 1.6 & 24 \\
\hline \multirow[t]{2}{*}{$\mathrm{E}$} & 0.9 & 8.1 & 60 & 90 & 230 & 2.98 & 3.08 & 4.15 & 15 & -86 & 10 & 19 \\
\hline & & & & & 345 & 2.96 & 3.04 & 6.07 & 15 & -82 & 9.7 & 24 \\
\hline \multirow[t]{2}{*}{$\mathrm{F}$} & 0 & 166.9 & 60 & 90 & 230 & 3.07 & 3.08 & 3.38 & 15 & -84 & 9.8 & 19 \\
\hline & & & & & 345 & 2.99 & 3.00 & 3.18 & 13 & -80 & 10 & 17 \\
\hline
\end{tabular}

Notes.

${ }^{a}$ Spin is given in units of the gravitational radius, $R_{G} \equiv G M c^{-2}=\frac{1}{2} R_{\mathrm{S}}$.

b Accretion disk major axis position angle (east of north).

${ }^{\mathrm{c}}$ Stokes $I$ flux density of integrated quiescent disk alone and minimum/maximum of system with orbiting hot spot.

d Polarization fraction and EVPA of the disk emission alone and minimum/maximum polarization fraction of system with orbiting hot spot.

\section{POLARIMETRIC CONSIDERATIONS}

For ideal circularly polarized feeds, the perfectly calibrated correlations are related to the complex Stokes visibilities $\left(I_{\nu}, Q_{\nu}, U_{\nu}, V_{\nu}\right)$ as follows:

$$
\begin{aligned}
& R R=I_{v}+V_{v} \\
& L L=I_{v}-V_{v} \\
& R L=Q_{v}+i U_{v} \\
& L R=Q_{v}-i U_{v},
\end{aligned}
$$

where $i=\sqrt{-1}$ and $R L$ (for example) denotes the right circular polarized signal at one station correlated against the left circular polarized signal at another. We have used the convention of Cotton (1993). Other definitions, differing in sign or rotation of the $R L$ and $L R$ terms by factors of $i$, are possible (e.g., Thompson et al. 2001), but do not affect the analysis. Significant circular polarization is neither predicted in the hot spot models nor observed at the resolution of connected-element arrays (Bower et al. 2003; Marrone et al. 2006a). In the limit of no circular polarization $\left(V_{v}=0\right), I_{v}=R R=L L$ is a direct observable in the parallel-hand correlations, but $Q_{\nu}$ and $U_{v}$ appear only in combination in the cross-hand correlations. $R L$ and $L R$ visibilities, which are direct observables, are constructed by appropriate complex addition of the Stokes $Q_{\nu}$ and $U_{\nu}$ visibilities. Right- and left-circular polarized (RCP and LCP) feeds are preferable to linearly polarized feeds for detecting linear polarization, since the latter mix Stokes $I_{\nu}$ with $Q_{v}$ in the parallel-hand correlations (Thompson et al. 2001).

For a point source, $I_{v} \geqslant \sqrt{Q_{v}^{2}+U_{v}^{2}+V_{v}^{2}}$. However, for an extended distribution, the polarized Stokes visibilities can exceed the amplitude of the Stokes $I_{v}$ visibility. (For instance, a uniform total intensity distribution with constant linear polarization fraction but a changing linear polarization angle will produce no power in Stokes $I_{\nu}$ on scales small compared to the distribution, but the Stokes visibilities $Q_{v}$ and $U_{v}$ will be nonzero.)

Analysis of polarimetric data is more complex than total intensity (Stokes $I$; we will henceforth drop the subscript on Stokes visibilities) data, but ratios of cross-hand ( $R L$ and
$L R)$ to parallel-hand $(R R$ and $L L)$ visibilities provide robust baseline-based observables immune to most errors arising from miscalibrated antenna complex gains. This stands in contrast to the single-polarization case in which robust observables can only be constructed from closure quantities on three or more telescopes. The procedure for referencing cross-hand data to parallel-hand data is explained in detail in Cotton (1993) and Roberts et al. (1994) and has been used successfully in experiments (e.g., Wardle 1971). Several details warrant further discussion. We shall refer to the full expressions for the observed correlation quantities:

$$
\begin{aligned}
R R=R_{1} R_{2}^{*}= & G_{1 R} G_{2 R}^{*}\left[\left(I_{12}+V_{12}\right) e^{i\left(-\varphi_{1}+\varphi_{2}\right)}\right. \\
& +D_{1 R} D_{2 R}^{*}\left(I_{12}-V_{12}\right) e^{i\left(+\varphi_{1}-\varphi_{2}\right)} \\
& +D_{1 R} P_{21}^{*} e^{i\left(+\varphi_{1}+\varphi_{2}\right)} \\
& \left.+D_{2 R}^{*} P_{12} e^{i\left(-\varphi_{1}-\varphi_{2}\right)}\right] \\
L L=L_{1} L_{2}^{*}= & G_{1 L} G_{2 L}^{*}\left[\left(I_{12}-V_{12}\right) e^{i\left(+\varphi_{1}-\varphi_{2}\right)}\right. \\
& +D_{1 L} D_{2 L}^{*}\left(I_{12}+V_{12}\right) e^{i\left(-\varphi_{1}+\varphi_{2}\right)} \\
& +D_{1 L} P_{12} e^{i\left(-\varphi_{1}-\varphi_{2}\right)} \\
& \left.+D_{2 L}^{*} P_{21}^{*} e^{i\left(+\varphi_{1}+\varphi_{2}\right)}\right], \\
R L=R_{1} L_{2}^{*}= & G_{1 R} G_{2 L}^{*}\left[P_{12} e^{i\left(-\varphi_{1}-\varphi_{2}\right)}\right. \\
& +D_{1 R} D_{2 L}^{*} P_{21}^{*} e^{i\left(+\varphi_{1}+\varphi_{2}\right)} \\
& +D_{1 R}\left(I_{12}-V_{12}\right) e^{i\left(+\varphi_{1}-\varphi_{2}\right)} \\
& \left.+D_{2 L}^{*}\left(I_{12}+V_{12}\right) e^{i\left(-\varphi_{1}+\varphi_{2}\right)}\right], \\
L R=L_{1} R_{2}^{*}= & G_{1 L} G_{2 R}^{*}\left[P_{21}^{*} e^{i\left(+\varphi_{1}+\varphi_{2}\right)}\right. \\
& +D_{1 L} D_{2 R}^{*} P_{12} e^{i\left(-\varphi_{1}-\varphi_{2}\right)} \\
& +D_{1 L}\left(I_{12}+V_{12}\right) e^{i\left(-\varphi_{1}+\varphi_{2}\right)} \\
& \left.+D_{2 R}^{*}\left(I_{12}-V_{12}\right) e^{i\left(+\varphi_{1}-\varphi_{2}\right)}\right],
\end{aligned}
$$

where numeric subscripts refer to antenna number, letter subscripts refer to the polarization (RCP or LCP), a star denotes complex conjugation, $G_{n X}=g_{n X} e^{i \psi_{n X}}$ is the complex gain in polarization $X \in\{R, L\}$ at antenna $n, P=Q+i U, D_{n X}$ is the instrumental polarization, and $\varphi_{n}$ is the parallactic angle (equations reproduced from Roberts et al. 1994). The $\varphi_{n}$ terms are constant for equatorial mount telescopes and can be incor- 
porated into the $G$ and $D$ terms, while for alt-azimuth mount telescopes the $\varphi_{n}$ terms vary predictably based on source declination, hour angle, and antenna latitude. It is likely that all of the telescopes in potential millimeter-wavelength VLBI arrays in the near future will have $\varphi_{n}$ terms varying with parallactic angle.

The ratio of cross-hand to parallel-hand data (e.g., $R L / L L$ ) contains an additional phase contribution $\Psi_{n}=\psi_{n R}-\psi_{n L}$ equal to the phase difference of the complex gains of the right and left circular polarizations of antenna $n$ (Brown et al. 1989). These phase differences also enter into closure phases of crosshand correlations as $\Psi_{1}+\Psi_{2}+\Psi_{3}$. Fortunately, the right-left phase differences vary slowly with time (e.g., Roberts et al. 1994), since the atmospheric transmission is not significantly birefringent at millimeter wavelengths and both polarizations are usually tied to the same local oscillator. We will henceforth assume that the $\Psi$ terms can be properly calibrated (for instance by observations of an unpolarized calibrator source), although proper calibration may not be strictly necessary for periodicity detection, since the expected timescale of variation of source structure is significantly faster than the timescale of variation of $\Psi$. Similarly, it is possible to determine the ratio of amplitudes of the real gains $\left(r_{n}=g_{n R} / g_{n L}\right)$ from observations of a suitable calibrator. In general, $r_{n}$ usually shows greater short-timescale variability than $\Psi_{n}$ (Roberts et al. 1994). Provided that proper instrumental polarization calibration is done, the fluctuation in $r_{n}$ can be estimated from the $R R / L L$ visibility ratio, since Sgr A* is expected to have no appreciable circular polarization. ${ }^{5}$ Even absent any complex gain calibration, it is probable that the contamination of the time series of cross-to-parallel amplitude ratios and (especially) phase differences by changes in $r_{n}$ and $\Psi_{n}$, respectively, will also be seen in the $R R / L L$ amplitude ratio and $R R-L L$ phase difference. Thus, large deviations seen in the cross-to-parallel quantities but not in the parallel-to-parallel quantities will likely be due to source structure differences, not gain miscalibration.

Correcting for instrumental polarization (the $D$-terms) may be more difficult. Effectively, the $D$-terms mix Stokes $I$ into the $R L$ and $L R$ terms (Thompson et al. 2001). Observations of calibrators with the Coordinated Millimeter VLBI Array (CMVA) at $\lambda=3.5 \mathrm{~mm}$ found $D$-terms ranging from a few to $21 \%$, with typical values slightly greater than $10 \%$ (Attridge 2001; Attridge et al. 2005). Polarimetric observations with CARMA and the SMA in their normal capacity as connectedelement interferometers have demonstrated that the instrumental polarization terms on some of the telescopes that will be included in future observations may be as low as a few percent (Bower et al. 2002; Marrone et al. 2006a, 2007). However, it is unknown how large the $D$-terms will be for potential VLBI arrays at $\lambda=1.3$ and $0.8 \mathrm{~mm}$, as many of the critical pieces of hardware (including feeds, phased-array processors, and even the antennas themselves) do not yet exist for some of the elements of such arrays. In any case, contributions from the $D$-terms may be comparable to or larger than contributions from the source polarization, at least on the shorter baselines. The timescale of variations of $D$-terms is typically much longer than the timescale on which the source structure in Sgr A* changes, so carefully designed observations may allow for the $D$-terms to be calibrated. At the angular resolution of the SMA, polarization

\footnotetext{
5 Stokes $V$ enters the expressions for $R R$ and $L L$ only as $I \pm V$, so even if circular polarization is detected, it will not prevent the estimation of $r_{n}$ unless the circular polarization fraction on angular scales accessible to VLBI is large or highly variable.
}

fractions of Sgr $\mathrm{A}^{*}$ at 230 and $345 \mathrm{GHz}$ range between $4 \%$ and $10 \%$ (Marrone et al. 2007), although the polarization fraction may exceed this range during a flare (Marrone et al. 2008). Linear polarization fractions derived from single-dish and connected-element millimeter observations of Sgr $\mathrm{A}^{*}$ are likely underestimates of the linear polarization fractions that will be seen with VLBI, since partial depolarization from spatially separated orthogonal polarization modes may occur when observed with insufficient angular resolution to separate them. That is, the small-scale structure that will be seen by VLBI is likely to have a larger polarization fraction than that observed so far with connected-element interferometry.

Calibration of the electric vector polarization angle (EVPA) may be difficult, at least in initial observations, due to the lack of known millimeter-wavelength polarization calibration sources (see, e.g., Attridge 2001). EVPA calibration will eventually be important for understanding the mechanism of linear polarization generation in $\mathrm{Sgr} \mathrm{A}^{*}$, assuming that the linear polarization can be unambiguously corrected for Faraday rotation. However, the ability of cross-hand correlation data to detect changes in the EVPA is unaffected by absolute EVPA calibration.

In the low signal-to-noise $(\mathrm{S} / \mathrm{N})$ regime, the ratio of visibility amplitudes can be a biased quantity. Visibility amplitudes are non-negative by definition, and the complex addition of a large noise vector to a small signal vector in the visibility plane will bias the visibility amplitude to higher values. Nevertheless, even biased visibility amplitudes may be of some utility in detecting changing polarization structure. Since the complex phase of noise is uniform random, phase differences are unbiased quantities.

\section{RESULTS}

\subsection{Baseline Visibility Ratios}

While lower-resolution observations of Sgr A* find polarization of less than $10 \%$, the effective fractional polarization on smaller scales can be much larger. Figure 2 shows the amplitudes of the $(u, v)$ data $^{6}$ that would be produced by a disk and persistent, unchanging orbiting hot spot with parameters as given in Model A at $230 \mathrm{GHz}$. The range in amplitudes reflects the changing flux density, both in total flux (i.e., the zero-spacing flux at $u=v=0$ ) as well as on smaller spatial scales, as would be sampled via VLBI. Both total power (Stokes $I$ ) and polarization signatures fall off with baseline length, but on average the fractional polarization increases with longer baselines, and the ratio of Stokes visibility amplitudes can exceed unity. All of our models produce much higher polarization fractions on small angular scales than at large angular scales, and all models except for Model $\mathrm{F}$ at $345 \mathrm{GHz}$ produce a substantial set of cross-to-parallel visibility amplitude ratios in excess of unity on angular scales of 40-80 $\mu$ as and smaller.

We henceforth focus on ratios of cross-to-parallel baseline visibilities (e.g., $R L / I$ ). Plots of the $R L-I$ phase difference ${ }^{7}$ are shown in Figures 3 and 4 for models at 230 and $345 \mathrm{GHz}$, respectively. At a total data rate of $16 \mathrm{Gbit} \mathrm{s}^{-1}$, nearly all baselines exhibit signatures of changing polarization structure. Due to the weak polarized signal on the longest baselines, a phased array of a subset of ALMA (Chile 10, in the nomenclature of

\footnotetext{
6 It is important not to confuse the antenna spacing parameters measured in wavelengths (conventionally denoted by lower-case $u$ and $v$ ) with the Stokes parameters (denoted by upper-case $U$ and $V$ ).

Note that $\arg (R L)-\arg (I)=\arg (R L / I)$.
} 


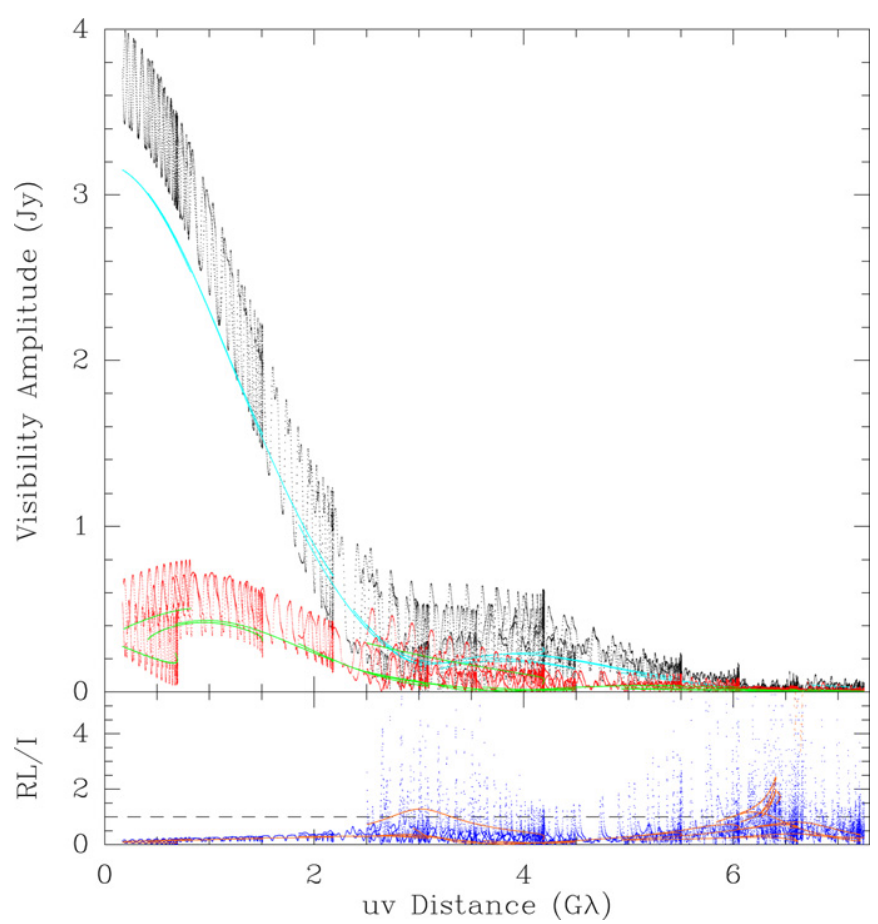

Figure 2. Top: visibility amplitude as a function of projected baseline length $\left(\sqrt{u^{2}+v^{2}}\right)$ for Model A at $230 \mathrm{GHz}$ (noiseless). Stokes $I$ is shown in black, and $R L$ is shown in red. A real orbiting hot spot would persist for only a small fraction of a day, producing a plot corresponding to a subset of the above points. Contributions from the disk alone in the absence of a hot spot are shown in cyan (Stokes $I$ ) and green $(R L)$. Bottom: ratio of $R L / I$ visibility amplitudes for the disk and hot spot (blue) and disk alone (orange). On small scales, $R L / I$ can greatly exceed unity.

(A color version of this figure is available in the online journal.)
Section 2) may be required in order to confidently detect polarization changes on the long baselines, especially to Europe. The PV-PdB baseline (and to a lesser extent the SMT-CARMA baseline at $230 \mathrm{GHz}$ ) effectively tracks the orientation of the total linear polarization, since Sgr A* is nearly unresolved on this short baseline, and the calibrated $R L$ phase of a polarized point source at phase center is twice the EVPA of the source (e.g., Cotton 1995).

Figures 5 and 6 show the $R L / I$ visibility amplitude ratio for selected baselines at 230 and $345 \mathrm{GHz}$, respectively. The shortest baselines, PV-PdB and SMT-CARMA, effectively track the large-scale polarization fraction as would be measured by the SMA, for instance. Because the short baselines resolve out several tens of percent of the total intensity emission (as compared to the zero-spacing flux in Figure 2) but a much smaller fraction of the polarized emission, the variation in the $R L / I$ and $L R / I$ amplitude ratios is fractionally larger than in the large-scale polarization fraction. A bias can be seen in the amplitude ratios when the $\mathrm{S} / \mathrm{N}$ is small, as noted in Section 3. (For brevity, we have shown only plots of the $R L-I$ phase difference and $R L / I$ amplitude ratio. The $L R-I$ phase difference and $L R / I$ amplitude ratio exhibit similar behavior.)

Closure phases of the cross-hand terms can be constructed in the same manner as for the parallel-hand terms, and these are robust observables. However, closure quantities are less necessary in the polarimetric case than for total-intensity observations because robust baseline-based observables can be constructed. As Figure 2 shows, the visibility amplitude in the cross-hand correlations is much lower than that of the parallel-hand correlations on short baselines. The $\mathrm{S} / \mathrm{N}$ of the closure phase is lower by a factor of $\sqrt{3}$ than the three constituent baseline $\mathrm{S} / \mathrm{Ns}$

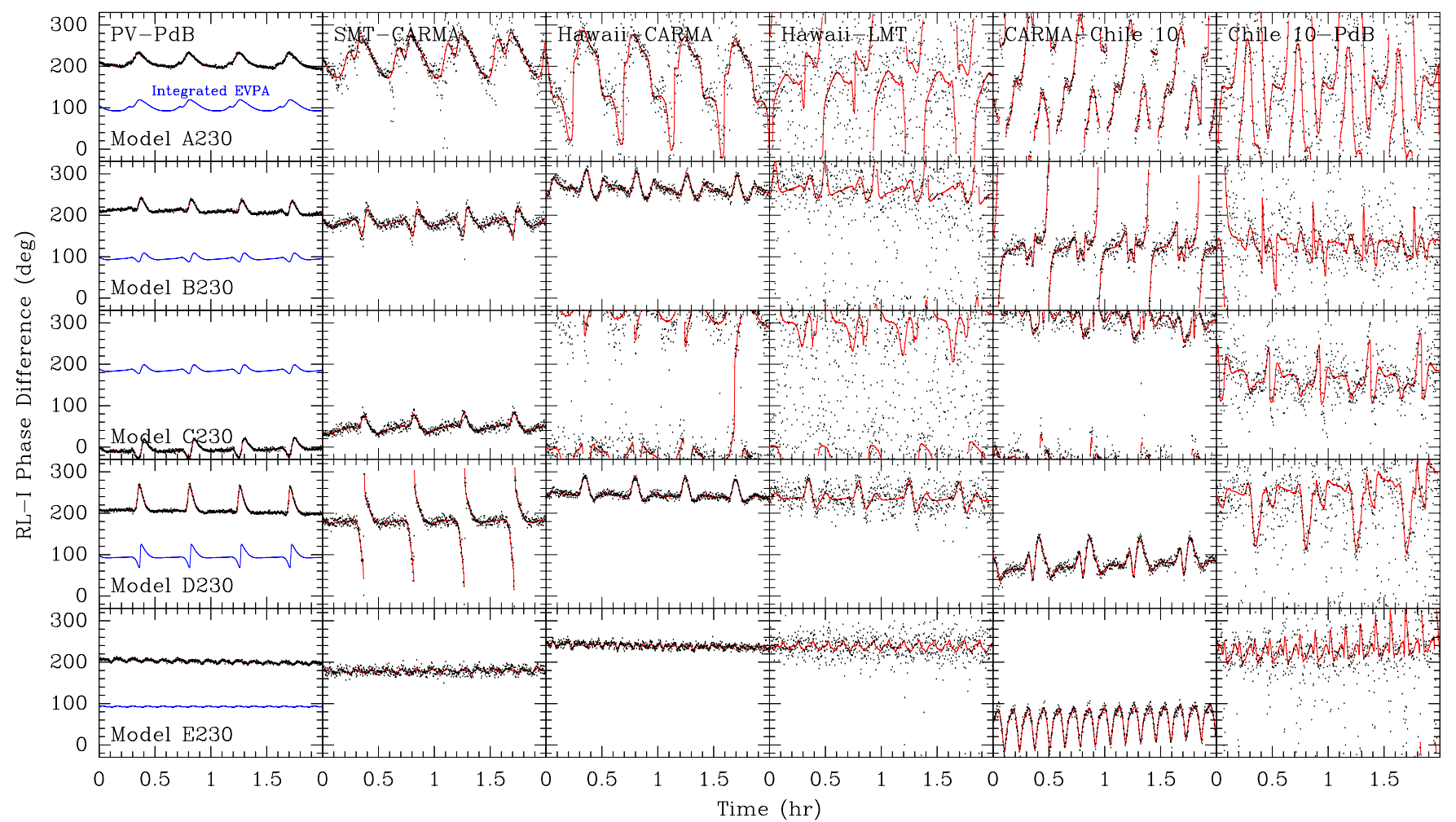

Figure 3. $R L-I$ phase differences for selected baselines at $230 \mathrm{GHz}$. The same $2 \mathrm{hr}$ of data, representing 4.5 periods (14.8 periods for Model E), are shown on all baselines except those involving PV or PdB. The solid line (red in the online version) indicates the expected signal in the absence of noise, and the dots indicate simulated data at $8 \mathrm{Gbit} \mathrm{s}^{-1}$ in each polarization $\left(16 \mathrm{Gbit} \mathrm{s}^{-1}\right.$ total). The blue line shows the EVPA that would be observed if the source were unresolved.

(A color version of this figure is available in the online journal.) 


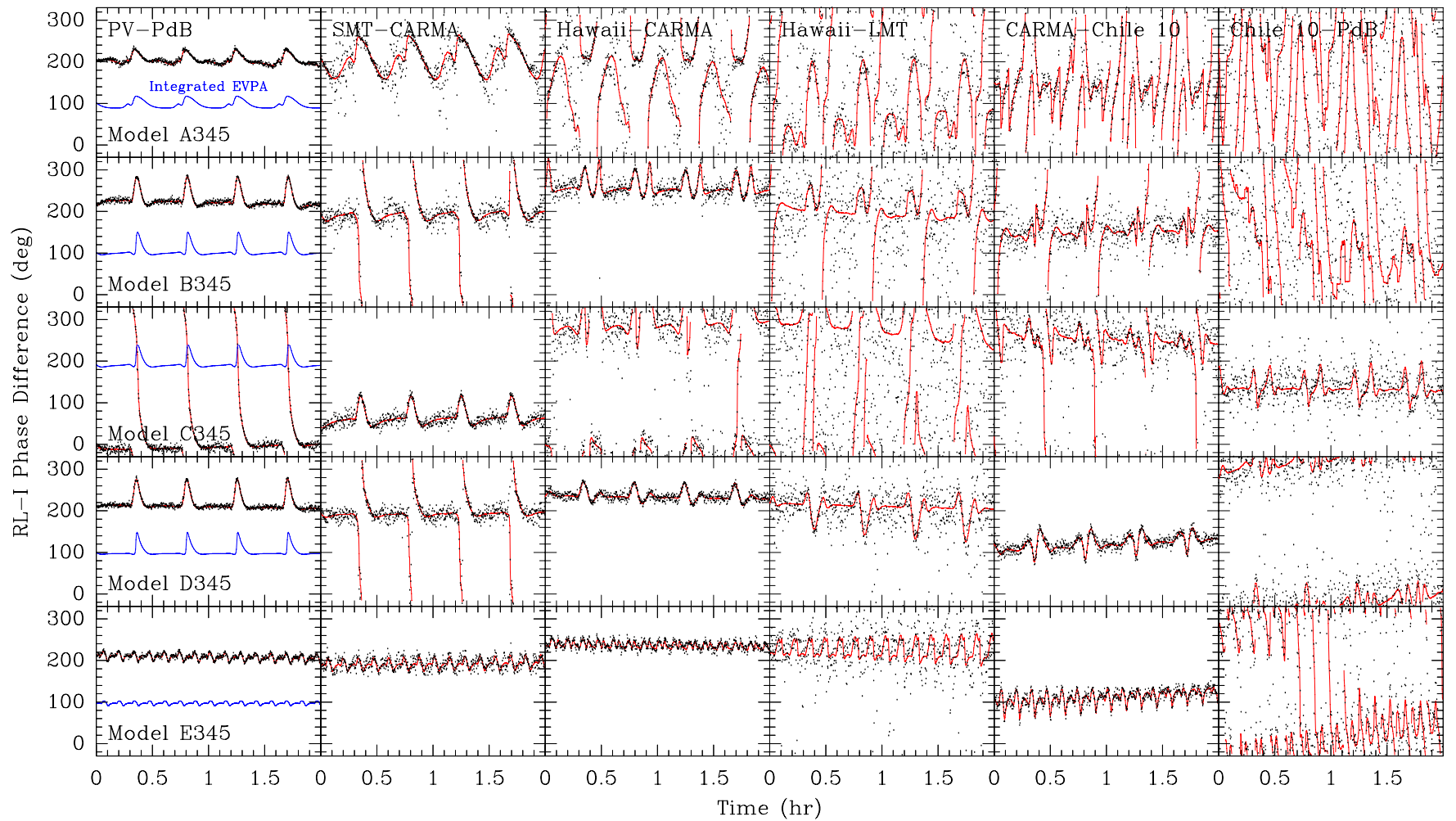

Figure 4. $R L-I$ phase differences for selected baselines at $345 \mathrm{GHz}$. See Figure 3 for details.

(A color version of this figure is available in the online journal.)

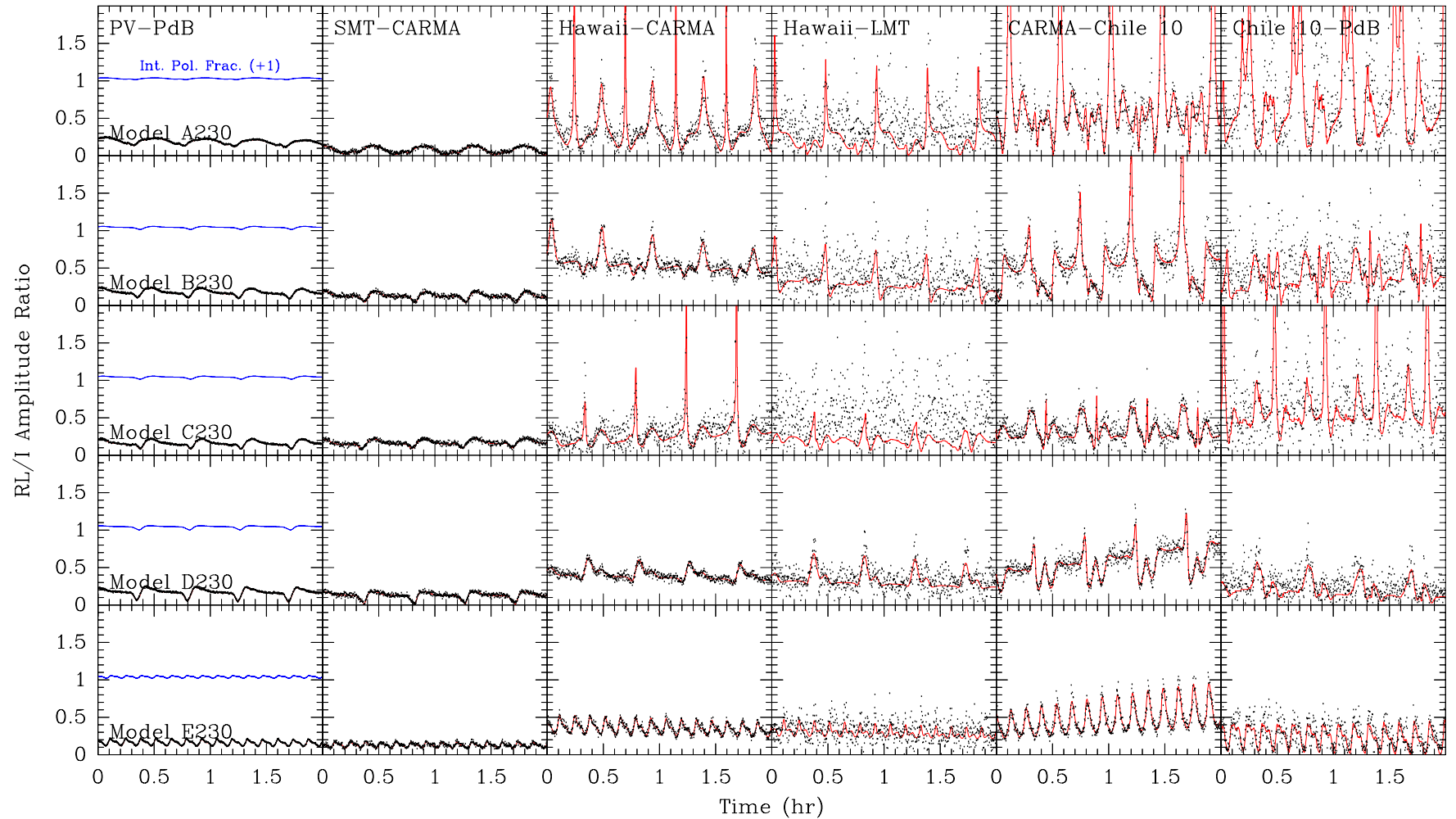

Figure 5. $R L / I$ amplitude ratio for selected baselines at $230 \mathrm{GHz}$. See Figure 3 for details. The blue line shows the integrated polarization fraction for the indicated model, shifted by 1.0 for clarity.

(A color version of this figure is available in the online journal.)

when the latter are all equal and is dominated by that of the weakest baseline when there is a large difference in the baseline S/Ns (Rogers et al. 1995). In Stokes I, the mean baseline S/Ns (averaged over multiple orbits) are greater than or equal to 5 on virtually all baselines and all models at $16 \mathrm{Gbit} \mathrm{s}^{-1}$ total bit rate ( $8 \mathrm{Gbit} \mathrm{s}^{-1}$ each RR and LL) in a $10 \mathrm{~s}$ coherence interval, pro- 


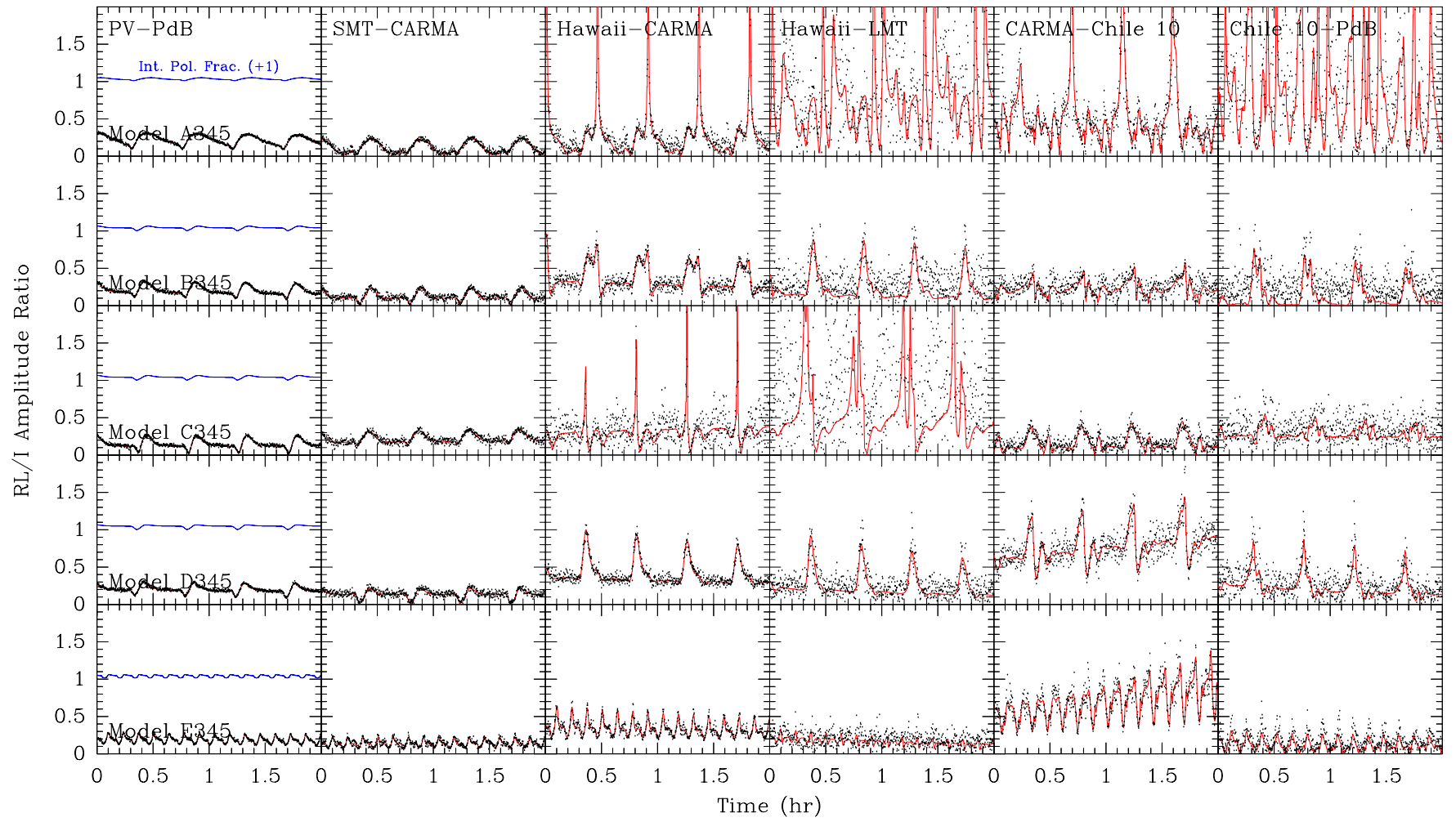

Figure 6. $R L / I$ amplitude ratio for selected baselines at $345 \mathrm{GHz}$. See Figures 3 and 5 for details.

(A color version of this figure is available in the online journal.)

vided that the Chile 10 is used in lieu of Chile 1 . The number of triangles with $\mathrm{S} / \mathrm{Ns}$ greater than 5 on all baselines at $8 \mathrm{Gbit} \mathrm{s}^{-1}$ in $R L$ or $L R$ is much smaller. Depending on the model, the SMT-CARMA-LMT and SMT/CARMA-LMT-Chile 10 triangles usually satisfy this condition, with Hawaii-SMT-CARMA also having sufficient $\mathrm{S} / \mathrm{N}$. Completion of the LMT, resulting in a system equivalent flux density of $\lesssim 600 \mathrm{Jy}$ at $230 \mathrm{GHz}$, will allow for strong detections on the Hawaii-LMT baseline and, importantly, significantly strengthen detections on the LMT-Chile baseline. If the coherence time is significantly shorter than $10 \mathrm{~s}$, or if the observed flare flux density is substantially lower than assumed in our models, closure phases may not have a large enough $\mathrm{S} / \mathrm{N}$ to detect periodic changes. In any case, if polarimetric visibility ratios are successful in detecting periodicity, there may not be a need to appeal to closure quantities except insofar as they can be used to improve the array calibration.

\subsection{Instrumental Polarization Calibration}

We have also simulated the effects of not correcting for parallactic angle terms and instrumental polarization by including Gaussian random $D$-terms of $11 \% \pm 3 \%$ with uniform random phases, based on the Attridge (2001) and Attridge et al. (2005) CMVA studies. This should be considered a worst-case scenario. $D$-terms at many of the telescopes will likely be substantially better: e.g., $1 \%-6 \%$ at the SMA in observations by Marrone et al. (2006a, 2007) and about 5\% at the $6 \mathrm{~m}$ antennas of the CARMA array (Bower et al. 2002). These quantities affect the observed correlation quantities $R R, L L, R L$, and $L R$ as indicated in Section 3. We have ignored terms of order $D^{2}$, but we have included terms of the form $D P$, since the polarized visibility amplitudes can be larger than the Stokes $I=\frac{1}{2}(R R+L L)$ visibility amplitudes on long baselines (Figure 2).
Example data showing the effects of large uncalibrated $D$ terms is shown in Figure 7. Instrumental polarization adds a bias to the ratio of cross-hand to parallel-hand visibility amplitudes (e.g., $R L / R R$ ) as well as a phase slope and offset to the difference of cross-hand and parallel-hand phases (e.g., $R L-R R)$. These effects are much more pronounced on the short baselines, especially PV-PdB and SMT-CARMA, because the fractional source polarization on large scales is small (and thus $|D I| \ll|P|$ ). In most cases, the cross-to-parallel amplitude ratios and phase differences behave similarly whether instrumental polarization calibration is included or not simply by virtue of the fact that the polarized intensity is a large fraction of the total intensity. Deviations in the cross-to-parallel phase difference response appear qualitatively large when the cross-hand amplitudes are near zero because small offsets from the source visibility, represented as a vector in the complex plane, can produce large changes in the angle (i.e., phase) of the visibility. Large instrumental polarization can affect the expected baseline-based signatures but do not obscure periodicity, since source structure changes in Stokes $I$ and $P$ have the same period in our models. Of course, proper $D$-term calibration is a sine qua non for modeling the polarized source structure (but not for detecting periodicity). The $D$-terms can be measured by observing a bright unpolarized calibrator (or polarized, unresolved calibrator), and the visibilities should be corrected for instrumental polarization if possible.

\subsection{Periodicity Detection}

As in Paper I, we can define autocorrelation functions to test for periodicity. More optimal methods exist to extract the period of a time series of data (Rogers et al. 2009), but the autocorrelation function is conceptually simple and suffices for our models. The amplitude autocorrelation function evaluated 

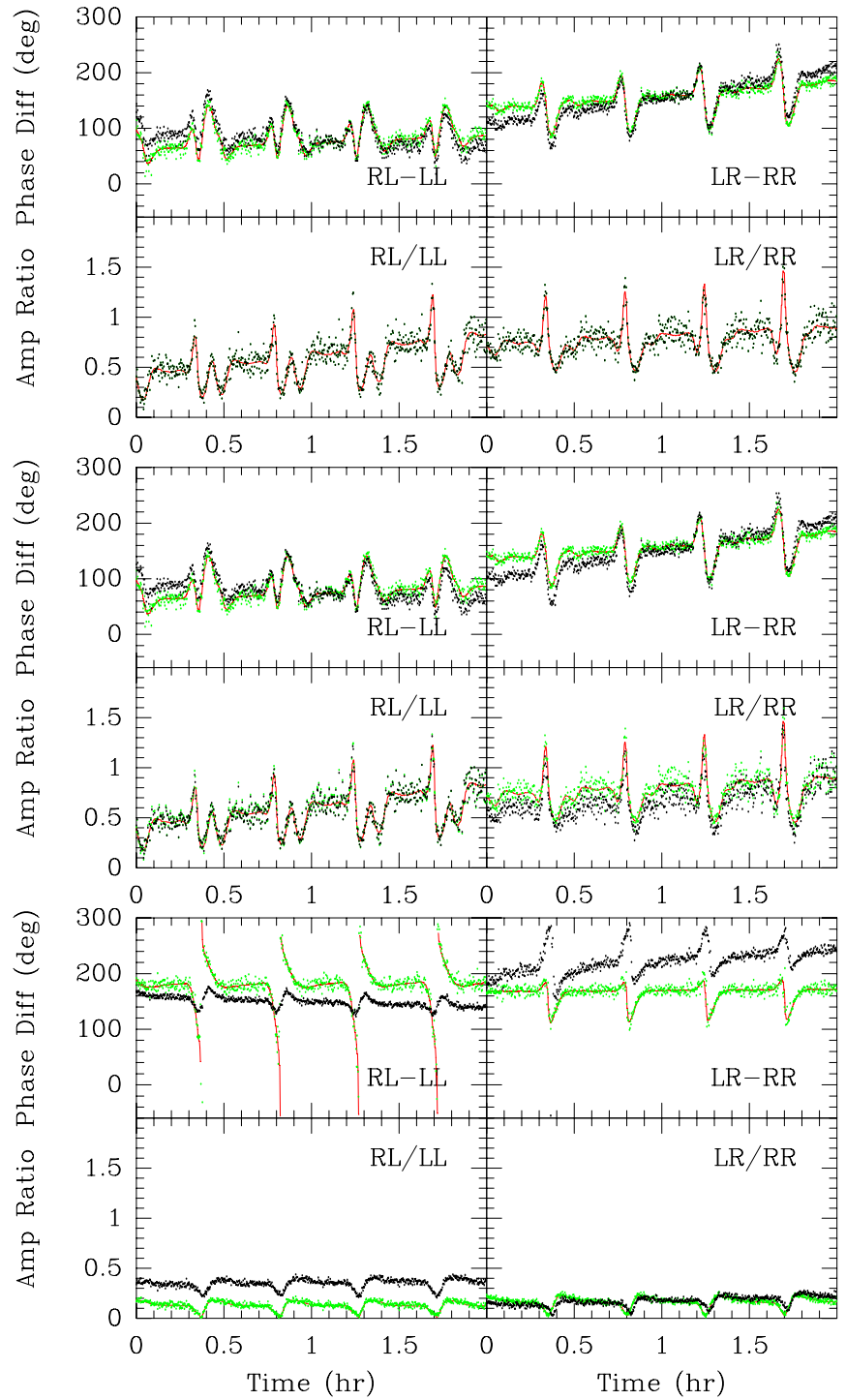

Figure 7. Example polarimetric visibility ratio quantities with and without parallactic angle and $D$-term calibration. Noiseless model values are shown in red, simulated data ( $8 \mathrm{Gbit} \mathrm{s}^{-1}$ per polarization) without parallactic angle and $D$-terms are shown in green, and simulated data with parallactic angle and $D$ terms are overplotted in black. Top: simulated data for the CARMA-Chile 10 baseline of Model D at $230 \mathrm{GHz}$ with zero $D$-terms. Parallactic angle terms effectively produce a small slope in the phase difference terms over timescales of interest and have no effect on visibility amplitudes in the absence of $D$ terms (i.e., the green and black dots are identical). Middle: the same baseline with $11 \% \pm 3 \% D$-terms. The inclusion of $D$-terms does not have a large effect because $|P|$ is several times larger than $|D I|$. Bottom: the SMT-CARMA baseline with $11 \% \pm 3 \% D$-terms. Uncalibrated $D$-terms will bias amplitude ratios and may produce qualitatively different phase differences when the crossto-parallel amplitude ratio is small but will not obscure periodicity even if the $D$-terms are large (as is assumed in this worst-case scenario based on the experience of Attridge (2001) and Attridge et al. (2005) at $86 \mathrm{GHz}$ ). The effect of uncalibrated $D$-terms will likely be largest on short baselines.

(A color version of this figure is available in the online journal.)

at lag $k$ on a time series of $n$ amplitude ratios $A_{i}=R L_{i} / I_{i}$ (or $\left.L R_{i} / I_{i}\right)$ on a baseline is defined as

$$
\mathrm{ACF}_{A}(k) \equiv \frac{1}{(n-k) \sigma^{2}} \sum_{i=1}^{n-k}\left[\left(\log A_{i}-\mu\right)\left(\log A_{i+k}-\mu\right)\right],
$$

where $\mu$ and $\sigma^{2}$ are the mean and variance of the logarithm of the amplitude ratios, respectively. The phase autocorrelation function is defined as

$$
\mathrm{ACF}_{\phi}(k) \equiv \frac{1}{n-k} \sum_{i=1}^{n-k} \cos \left(\phi_{i}-\phi_{i+k}\right),
$$

where $\phi_{i}$ denotes the $R L-I$ or $L R-I$ phase difference of point $i$. By definition, $\mathrm{ACF}_{A}(0)=\mathrm{ACF}_{\phi}(0)=1$. The largest non-trivial peak corresponds to the period, with the caveat that the changing baseline geometries caused by Earth rotation can conspire to cause the autocorrelation function to be slightly greater at integer multiples of the true period. The phase autocorrelation function can suffer from lack of contrast when the visibility phase difference is not highly variable as may be the case for the shortest baselines depending on the model (Figure 8), but the lack of contrast is not so severe as in the total-intensity case (cf. Paper I) due to the sensitivity of short-baseline cross-to-parallel phase differences to the source-integrated EVPA.

An array consisting of Hawaii, SMT, and CARMA is sufficient to confidently detect periodicity at a total bit rate of 2 Gbit $\mathrm{s}^{-1}$ (i.e., $0.25 \mathrm{GHz}$ bandwidth per polarization) over 4.5 orbits of data for Models A-E. This contrasts with the total intensity case, in which a substantially higher bit rate is required on the same array, depending on the model (Paper I). The key point is that the cross-to-parallel visibility ratios on the short baselines trace the overall source polarization fraction and EVPA, which are readily apparent even at the much coarser angular resolution afforded by connected-element interferometry (e.g., Marrone et al. 2006b). Long baselines are thus not strictly necessary to detect periodic polarimetric structural changes, although they will be important for modeling the small-scale polarimetric structure of the Sgr A*. In contrast, significantly higher bit rates and 4-element arrays are usually required to detect periodic source structure changes in total intensity (Paper I).

Long-period models (e.g., Model F) are problematic for millimeter VLBI periodicity detection because it may not be possible to detect more than two full periods during the window of mutual visibility between most of the telescopes in a potential VLBI array. As with the total intensity case (Paper I), the most promising approach for millimeter VLBI is to observe with an array of four or five telescopes, since large changes in cross-toparallel phase differences and visibility amplitude ratios tend to be episodic across most or all baselines. The LMT is usefully placed because it provides a long window of mutual visibility to Chile and the US telescopes as well as a small time overlap with PV, thus enabling a large continuous time range over which Sgr A* is observed. Assuming that the flare source can survive for several orbital periods, connected-element interferometry may suffice to demonstrate periodicity. Sgr A* is above $10^{\circ}$ elevation for approximately $9 \mathrm{hr}$ from Hawaii and $12 \mathrm{hr}$ from Chile.

\section{DISCUSSION}

\subsection{Faraday Rotation and Depolarization}

VLBI polarimetry has several key advantages over single-dish and connected-element interferometry for understanding the polarization properties of Sgr A*. First, even the shortest baselines likely to be included in the array will filter out surrounding emission. Reliable single-dish extraction of polarization information requires subtracting the contribution from the surrounding dust, which can dominate the total polarized flux at $345 \mathrm{GHz}$ and is significant even at $230 \mathrm{GHz}$ (Aitken et al. 2000). Contamination by surrounding emission is much less severe for SMA measurements, where the synthesized beamsize is on the order of an 


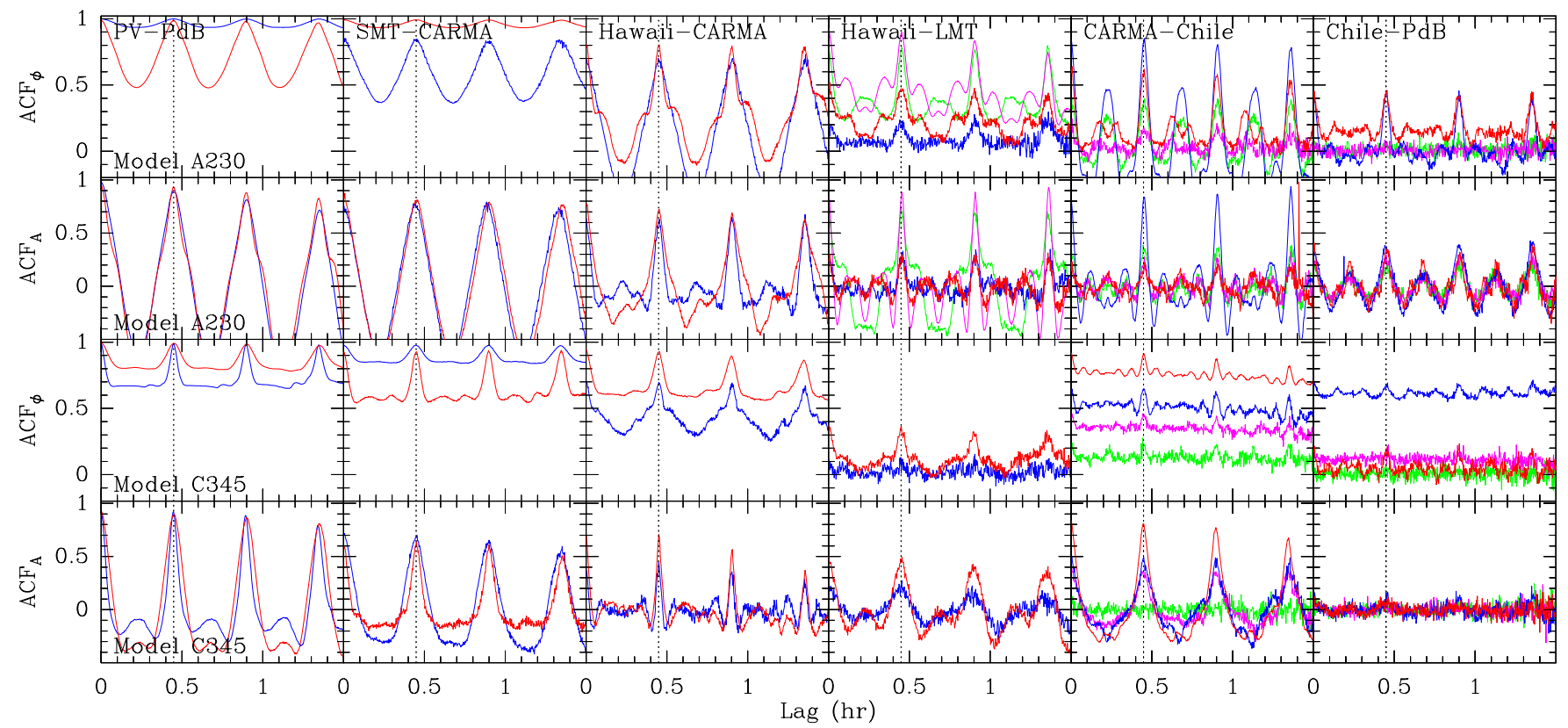

Figure 8. Autocorrelation functions for models $\mathrm{A} 230$ and $\mathrm{C} 345$ on selected baselines. Blue and red denote $R L / I$ and $L R / I$, respectively. Green and magenta indicate $R L / I$ and $L R / I$, respectively, using a completed $50 \mathrm{~m}$ LMT optimized for $230 \mathrm{GHz}$ performance (fourth column) or Chile 1 (in place of Chile 10 in the fifth and sixth columns). The dashed line indicates the orbital period.

(A color version of this figure is available in the online journal.)

arcsecond, depending on configuration (for instance, 1 1". $4 \times 2$ ".2 in the observations of Marrone et al. 2006a). VLBI will do much better still, with the shortest baselines resolving out most of the emission on scales larger than $\sim 1$ mas $\left(100 R_{S}\right)$, effectively restricting sensitivity to the inner accretion disk and/or outflow region.

Second, the resolution provided by millimeter VLBI will greatly reduce depolarization due to blending of emission from regions with different linear polarization directions. Models of the accretion flow predict that linear polarization position angles and Faraday rotation will be nonuniform throughout the source (Bromley et al. 2001; Broderick \& Loeb 2005, 2006; Huang et al. 2008). For this reason, ratios of cross-hand to parallel-hand visibilities (which are the visibility analogs of linear polarization fractions) can greatly exceed the total linear polarization fraction integrated over the source (compare Figure 2 and Table 1).

Third, VLBI polarimetry has the potential to identify whether changes in detected polarization are due to intrinsic source variability or changes in the rotation measure at larger distances. The former would be expected to be variable on relatively short timescales (minutes to tens of minutes), consistent with the orbital period of emission at a few gravitational radii. The latter would be expected to vary more slowly and affect only the polarized emission, not the total intensity. A cross-correlation between polarized and total-intensity data may allow the two effects to be disentangled.

Linear polarization at millimeter wavelengths can be used to estimate the accretion rate of Sgr A* (e.g., Quataert \& Gruzinov 2000). At frequencies below $\sim 100 \mathrm{GHz}$, no linear polarization is detected due to Faraday depolarization in the accretion region (Bower et al. 1999a, 1999b). Linear polarization is detected toward Sgr A* at higher frequencies (e.g., Aitken et al. 2000), where the effects of Faraday rotation are smaller. Ultimately, accretion rates are constrained by the lack of linear polarization at long wavelengths and its existence at short wavelengths. Measurements of the Faraday rotation exist, although it is unclear whether changes in detected polarization angles are due to changing source polarization structure or a variable rotation measure (Bower et al. 2005; Marrone et al. 2006a, 2007).

Longer term, imaging may be possible if all seven millimeter telescope sites heretofore considered (and possibly others as well) are used together as a global VLBI array (e.g., an Event Horizon Telescope; Doeleman et al. 2009b). Imaging the quiescent polarization structure of Sgr A* may allow the characteristics of the source emission region to be distinguished from those of the region producing Faraday rotation (which may overlap or be identical with the emission region). Contemporaneous millimeter VLBI observations at two different frequencies would allow separate maps of the intrinsic polarization structure and the rotation measure to be produced. It may also then be possible to place strong constraints on the density $\left(n_{e}\right)$ and magnetic fields $(B)$ in Sgr A*. Briefly, the rotation measure is related to $\int n_{e} B_{\|} d l$, while the total intensity is proportional to $\int n_{e} B_{\perp}^{\alpha+1} d l$, where $\alpha$ is the optically thin spectral index (Westfold 1959). Obtaining these results will require the ability to fully calibrate the data for instrumental polarization terms and the absolute EVPA. It may also require higher image fidelity than a seven-telescope VLBI array can provide (Fish \& Doeleman 2009). There are possibilities for extending a millimeter VLBI array beyond these seven sites by adding other existing (e.g., the South Pole Telescope) or new telescopes (Doeleman et al. 2009b), but full consideration of the scientific impact of potential future arrays is beyond the scope of this work.

\subsection{Physical Considerations}

The inclusion of a screen of constant Faraday rotation alters the phases of the cross-hand terms (and therefore the cross-toparallel phase differences) but does not materially affect the detectability of changing polarization structure. The mean rotation measure of Sgr A* averaged over multiple epochs is $(-5.6 \pm 0.7) \times 10^{5} \mathrm{rad} \mathrm{m}^{-2}$ (Marrone et al. 2007), which 
corresponds to a rotation of polarization vectors by $-55^{\circ}$ at $230 \mathrm{GHz}$ and $-24^{\circ}$ at $345 \mathrm{GHz}$. Persistent gradients of rotation measure across the source are virtually indistinguishable from intrinsic polarization structure in the case of a steady-state source, but it is possible that the source structure and rotation measure change on different timescales, which would allow the two effects to be disentangled (Marrone et al. 2007). Comparison of changes in the polarization data with total intensity data (obtained from the parallel-hand correlations) and total polarization fraction (obtained from simultaneous connected-element interferometric data if available, else inferred from the shortest VLBI baselines) may be useful for identifying whether observed polarization angle changes are due to a variable rotation measure (Bower et al. 2005).

The physical mechanism that produces flares in total intensity and polarization changes is poorly understood. Connectedelement interferometry at millimeter wavelengths has not been conclusive as to whether orbiting hot spots are the underlying mechanism that produces flares in Sgr A* (compare Marrone et al. 2006b and Marrone et al. 2008), or even as to whether multiple mechanisms may be responsible for flaring. Polarization variability can be decorrelated from total intensity variability (e.g., Marrone et al. 2006b), and each shows variability on timescales ranging from tens of minutes to hours (and possibly longer). Spatial resolution will be key to deciphering the environment of $\mathrm{Sgr} \mathrm{A}^{*}$, and thus there is a critical need for polarimetric millimeter-wavelength VLBI.

Our results are generalizable to any mechanism producing changes in linear polarization, whether due to orbiting or spiraling hot spots, jets, disk instabilities, or any other mechanism in the inner disk of Sgr A*. Visibility ratios on baselines available for millimeter-wavelength VLBI will provide reasonably robust observables to detect changes in the polarization structure on relevant scales from a few to a few hundred $R_{\mathrm{S}}$, regardless of the cause of those changes. Clearly, periodicity can only be detected if the underlying mechanism that produces polarization changes is itself periodic, but baseline visibility ratios will be sensitive to any changes that are rapid compared to the rotation of the Earth.

\subsection{Observational Strategy}

Future millimeter-wavelength VLBI observations of Sgr A* should clearly be observed in dual-polarization unless not allowed by telescope limitations. Total-intensity analysis via closure quantities, as outlined in Paper I, can be performed regardless of whether the data are taken in single- or dual-polarization mode, but the cross-hand correlations can only be obtained from dual-polarization data. The cross-hand correlation data provide additional chances to detect variability via changing source polarization structure.

By virtue of its size and location, which produces mediumlength baselines to Chile and Hawaii as well as a long window of mutual visibility with Chile, the LMT is a very useful telescope. The sensitivities assumed in this work for first light on the LMT may lead to biased amplitude ratios on the Hawaii-LMT baseline (Figure 5), but this will not prevent detection of periodicity (Figure 8). Thus, strong consideration should be given in favor of including the LMT in a millimeter-wavelength VLBI array observing Sgr A* as soon as possible. If the parameters of the fully completed LMT are assumed, the bias disappears and the scatter of points on the Hawaii-LMT baseline in Figure 5 is similar to that seen on the shorter Hawaii-CARMA baseline, and baselines between continental North America and the LMT will be of comparably good $\mathrm{S} / \mathrm{N}$ to the lower-resolution PVPdB baseline. Eventually, the LMT and ALMA will be the most sensitive stations in a millimeter VLBI array and will enable sensitive modeling of the Sgr A* system.

If possible, it would be advantageous to obtain connectedelement interferometric data of Sgr A* simultaneously with VLBI data. While amplitude ratios and phase differences on the PV-PdB and (to a lesser extent) SMT-CARMA baselines track the large-scale polarization fraction and EVPA fairly well in these models, it is not known what fraction of the polarization structure arises from larger-scale emission in Sgr A*. If interferometer stations can be configured to produce both crosscorrelations between telescopes as well as a phased output of all telescopes together, opportunities for simultaneous connectedelement interferometry may exist with the PdB Interferometer, the SMA, CARMA, or ALMA. If system limitations prevent this, it may still be possible to acquire very-short-spacing data with those telescopes in CARMA or ALMA that are not phased together for VLBI.

\section{CONCLUSIONS}

(Sub)millimeter-wavelength VLBI polarimetry is a very valuable diagnostic of emission processes and dynamics near the event horizon of Sgr $\mathrm{A}^{*}$. We summarize the findings in this paper as follows.

1. Millimeter-wavelength polarimetric VLBI can detect changing source structures. Despite low polarization fractions seen with connected-element interferometry, the much higher angular resolution data provided by VLBI will be far less affected by beam depolarization and contamination from dust polarization. Polarimetric VLBI provides an orthogonal way to detect periodic structural changes as compared with total intensity VLBI.

2. Ratios of cross- to parallel-hand visibilities are robust baseline-based observables. Short VLBI baselines approximately trace the integrated polarization fraction and position angle of the inner accretion flow of $\mathrm{Sgr} \mathrm{A}^{*}$, while longer VLBI baselines resolve smaller structures.

3. Calibration of instrumental polarization terms is not necessary to detect a changing source structure, including periodicity, in Sgr A*.

4. Polarimetric VLBI may be able to disentangle the effects of rotation measure from intrinsic source polarization. Initial results will likely come from observations of the timescale of polarimetric variability. If the initial array is expanded to allow high-fidelity imaging, polarimetric VLBI may be able to map the Faraday rotation region and directly infer the density and magnetic field structure of the emitting region in Sgr A*.

The high-frequency VLBI program at Haystack Observatory is funded through a grant from the National Science Foundation.

\section{REFERENCES}

Aitken, D. K., Greaves, J., Chrysostomou, A., Jenness, T., Holland, W., Hough, J. H., Pierce-Price, D., \& Richer, J. 2000, ApJ, 534, L173

Attridge, J. M. 2001, ApJ, 553, L31

Attridge, J. M., Wardle, J. F. C., \& Homan, D. C. 2005, ApJ, 633, L85

Bower, G. C., Backer, D. C., Zhao, J.-H., Goss, M., \& Falcke, H. 1999a, ApJ, 521,582

Bower, G. C., Falcke, H., Wright, M. C. H., \& Backer, D. C. 2005, ApJ, 618, L29 
Bower, G. C., Wright, M. C.H., Backer, D. C., \& Falcke, H. 1999b, ApJ, 527 851

Bower, G. C., Wright, M. C. H., Falcke, H., \& Backer, D. C. 2003, ApJ, 588, 331

Bower, G. C., Wright, M. C. H., \& Forster, R. 2002, Polarization Stability of the BIMA Array at $1.3 \mathrm{~mm}$, BIMA Memo 89

Broderick, A., \& Blandford, R. 2004, MNRAS, 349, 994

Broderick, A. E., \& Loeb, A. 2005, MNRAS, 363, 353

Broderick, A. E., \& Loeb, A. 2006, MNRAS, 367, 905

Bromley, B. C., Melia, F., \& Liu, S. 2001, ApJ, 555, L83

Brown, L. F., Roberts, D. H., \& Wardle, J. F. C. 1989, AJ, 97, 1522

Cotton, W. D. 1993, AJ, 106, 1241

Cotton, W. D. 1995, in ASP Conf. Ser. 82, Very Long Baseline Interferometry and the VLBA, ed. J. A. Zensus, P. J. Diamond, \& P. J. Napier (San Francisco, CA: ASP), 289

Doeleman, S. S., Fish, V. L., Broderick, A. E., Loeb, A., \& Rogers, A. E. E. 2009a, ApJ, 695, 59

Doeleman, S. S., et al. 2008, Nature, 455, 78

Doeleman, S. S., et al. 2009b, Astronomy, 2010, 68

Fish, V. L., \& Doeleman, S. S. 2009, in IAU Symp. 261, Relativity in Fundamental Astronomy: Dynamics, Reference Frames, and Data Analysis, ed. S. Klioner, P. K. Seidelmann, \& M. Soffel (Cambridge: Cambridge Univ. Press), in press (arXiv:0906.4040)

Huang, L., Liu, S., Shen, Z.-Q., Cai, M. J., Li, H., \& Fryer, C. L. 2008, ApJ, 676, L119
Huang, L., Liu, S., Shen, Z.-Q., Yuan, Y.-F., Cai, M. J., Li, H., \& Fryer, C. L. 2009, ApJ, 703, 557

Jones, T. W., \& O’Dell, S. L. 1977, ApJ, 214, 522

Macquart, J.-P., Bower, G. C., Wright, M. C. H., Backer, D. C., \& Falcke, H. 2006, ApJ, 646, L111

Markoff, S., Bower, G. C., \& Falcke, H. 2007, MNRAS, 379, 1519

Marrone, D. P., Moran, J. M., Zhao, J.-H., \& Rao, R. 2006a, ApJ, 640, 308

Marrone, D. P., Moran, J. M., Zhao, J.-H., \& Rao, R. 2006b, J. Phys. Conf. Ser., 54,354

Marrone, D. P., Moran, J. M., Zhao, J.-H., \& Rao, R. 2007, ApJ, 654, L57

Marrone, D. P., et al. 2008, ApJ, 682, 373

Petrosian, V., \& McTiernan, J. M. 1983, Phys. Fluids, 26, 3023

Quataert, E., \& Gruzinov, A. 2000, ApJ, 545, 842

Roberts, D. H., Wardle, J. F. C., \& Brown, L. F. 1994, ApJ, 427, 718

Rogers, A. E. E., Doeleman, S. S., \& Fish, V. L. 2009, BAAS, 41, 217

Rogers, A. E. E., Doeleman, S. S., \& Moran, J. M. 1995, AJ, 109, 1391

Rogers, A. E. E., et al. 1974, ApJ, 193, 293

Thompson, A. R., Moran, J. M., \& Swenson, G. W., Jr. 2001, Interferometry and Synthesis in Radio Astronomy (2nd ed.; New York: Wiley)

Trippe, S., Paumard, T., Ott, T., Gillessen, S., Eisenhauer, F., Martins, F., \& Genzel, R. 2007, MNRAS, 375, 764

Wardle, J. F. C. 1971, Astrophys. Lett., 8, 53

Westfold, K. C. 1959, ApJ, 130, 241

Yuan, F., Quataert, E., \& Narayan, R. 2003, ApJ, 598, 301 